\title{
Two-axis accelerometer based on multicore fibre Bragg gratings
}

\author{
Amanda Fender ${ }^{\mathrm{a}}$, William N. MacPherson ${ }^{\mathrm{a}}$, Robert R. J. Maier ${ }^{\mathrm{a}}$, James S. Barton ${ }^{\mathrm{a}}$, \\ David S. George ${ }^{\mathrm{b}}$, Robert I. Howden ${ }^{\mathrm{b}}$, Graham W. Smith ${ }^{\mathrm{b}}$, Ben J.S. Jones ${ }^{\mathrm{b}}$, Scott McCulloch ${ }^{\mathrm{b}}$, \\ Xianfeng Chen ${ }^{c}$, Rui Suo ${ }^{c}$, Lin Zhang ${ }^{c}$, Ian Bennion ${ }^{c}$ \\ ${ }^{a}$ Applied Optics and Photonics, Heriot-Watt University, Edinburgh, EH14 4AS, UK \\ ${ }^{\mathrm{b}}$ AWE Plc, Aldermaston, Reading, RG7 4PR, UK \\ ${ }^{c}$ Photonics Research Group, Aston University, Birmingham B4 7ET, UK
}

\begin{abstract}
We report an accelerometer based upon a simple fibre cantilever constructed from a short length of multicore fibre (MCF) containing fibre Bragg gratings (FBGs). Two-axis measurement is demonstrated up to $3 \mathrm{kHz}$.
\end{abstract}

Keywords: accelerometer, multicore fibre, fibre Bragg grating

\section{INTRODUCTION}

We have previously demonstrated two-axis curvature measurement using multicore fibre ${ }^{1}$, comprising a four core silica fibre with cores positioned at the vertices of a 50 micron square (fig 1). FBGs were written into each core at the same location along the fibre length and at nominally the same wavelength. These gratings enable the differential strain across the fibre to be measured, and can be interrogated using low bandwidth schemes (i.e optical spectrum analyser). For high speed measurement $(\mathrm{kHz})$ each FBG was interrogated by two channels of an arrayed waveguide grating (AWG) (fig 2), acting as bandpass filters where the ratio of power in each channel is a function of the grating wavelength. Interrogation speed of $10 \mathrm{~s}$ of $\mathrm{kHz}$ may be achieved with this method.

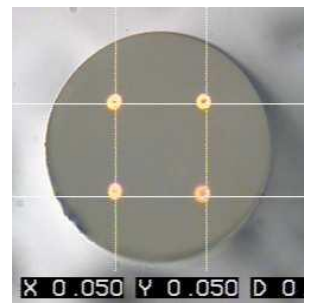

Fig 1: Cross section of multicore fibre

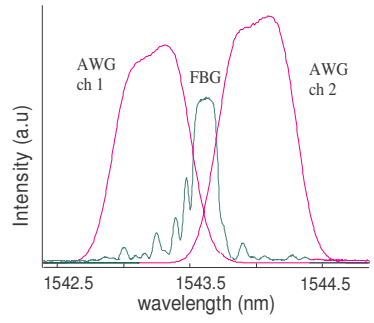

Fig 2: interrogation of MCF FBG by AWG

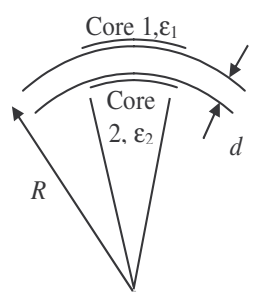

Fig 3: MCF curvature measurement

Curvature at the gratings may be inferred from the differential strain (fig 3). The difference between the Bragg shifts of two gratings is proportional to the curvature in the plane containing the cores. By using orthogonal pairs of cores, twoaxis curvature measurement can be realised. As this is a differential measurement, the sensor is insensitive to common mode temperature effects. AWG interrogation has been shown to give a curvature resolution of $0.09 \mathrm{~m}^{-1}$ at $>1 \mathrm{kHz}$.

Dynamic curvature measurement can form the basis of a fibre accelerometer. Dinev ${ }^{2}$ describes a two-dimensional accelerometer using an optical lever and a linear position photodetector, but this design requires electrical connection to the detector within the sensor head. A cantilever may be constructed by clamping a short length of fibre at one end. From cantilever theory its curvature is proportional to its transverse acceleration ${ }^{3}$. Single axis accelerometers based on this principle have commonly been intensity based sensors ${ }^{4}$, which are affected by any loss of signal in the system. Dependence on a fixed input intensity may be reduced by a ratio of two output fibres ${ }^{5,6}$. Single FBG based accelerometers require temperature compensation ${ }^{7,8}$. Temperature sensitivity of FBG accelerometers may be reduced by measuring the width of a chirped grating instead of the shift of an $\mathrm{FBG}^{9}$. By forming a cantilever from MCF with FBGs

*a.fender@hw.ac.uk

Third European Workshop on Optical Fibre Sensors, Antonello Cutolo, Brian Culshaw, José Miguel López-Higuera, Eds., Proceedings of SPIE Vol. 6619, 66190Q, (2007) $\cdot 0277-786 X / 07 / \$ 18 \cdot$ doi: 10.1117/12.738411 
written into each core, a two-axis, temperature insensitive accelerometer may be constructed. Using this technique as a basis for acceleration measurement is attractive for niche applications where electrical pickup is a problem.

\section{ACCELEROMETER CONCEPT AND DESIGN}

The accelerometer is formed by a short length of MCF with an end mass to increase sensitivity, and FBGs located next to the clamp, the region of maximum curvature due to bending. Figure 4 shows the configuration of the sensor.

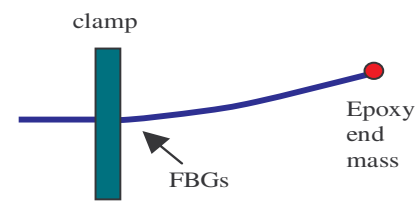

Fig 4 : Basic concept of mass loaded multicore fibre cantilever for acceleration measurement

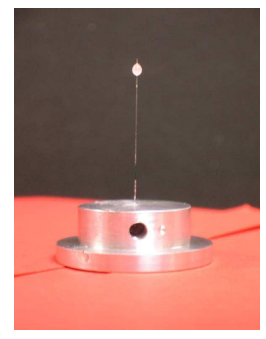

Fig 5 : Sensing element: fibre cantilever with epoxy end mass

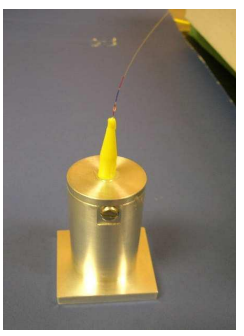

Fig 6 : Sensing element glued inside housing

The acceleration, $a$, of the MCF cantilever is related to the curvature $1 / R$ of the fibre at a distance $x$ from the clamp by the relationship shown in equation (1) where $m$ is the end mass, $E$ is Young's modulus of the fibre and $I$ is the second moment of area. The curvature of the fibre at the gratings' location is also proportional to the difference in Bragg shifts of the two sensing MCF gratings. Therefore a measure of the acceleration of the fibre may be obtained by measuring the FBG shifts in each core due to acceleration induced curvature.

$$
\mathrm{a}=\frac{\mathrm{EI}}{\mathrm{m}(\mathrm{x}-\mathrm{l})}\left(\frac{1}{\mathrm{R}}\right)
$$

$$
\mathrm{f} \propto \sqrt{\frac{3 \mathrm{EI}}{\mathrm{ml}^{3}}}
$$

Equation (1) shows that the acceleration range of the MCF accelerometer can be controlled to some extent by choosing the length of the cantilever and the mass applied to the end. The other parameters in this relationship are intrinsic fibre properties. The cantilever length and end mass also dictate the frequency response of the accelerometer (equation (2)).

The active part of the sensor - the MCF and end mass - only requires a simple support to allow it to be attached to the test structure. However to protect the fibre from damage the active length of fibre was enclosed in a small aluminium housing. The fibre was glued into a simple collar (figure 5) with the gratings positioned just outside the collar. The collar was then attached to the end of the main sensor housing. The final assembly of the accelerometer is shown in Figure 6.

\section{DYNAMIC TESTING}

\subsection{Frequency response}

To obtain the frequency response of each sensor, the accelerometer housing was fixed to a shaker and rotated to the correct orientation. The signal generator driving the shaker was set to frequencies between 40 and $3000 \mathrm{~Hz}$ and at each frequency the vibration amplitude was adjusted to give an acceleration of $2 \mathrm{~g}$, monitored using a piezoelectric reference accelerometer. At each frequency, the FBG shifts and the reference accelerometer were recorded. The MCF FBGs were interrogated by AWGs at $10 \mathrm{kHz}$. The sensor response in the vibration axis was then defined as the difference between wavelength shifts in the two cores of MCF aligned with the axis of vibration.

Figure 8 (top) shows the vibration axis sensor response, normalised to $1 \mathrm{~g}$ reference amplitude for three fibre cantilevers of length $24.95 \mathrm{~mm}$, with end masses $0.55,1.86$ and $4.82 \mathrm{mg}$, for sensors 1 to 3 respectively. Each of these sensors exhibit three natural frequencies in the range 0 to $3 \mathrm{kHz}$. There are also peaks at $\sim 200 \mathrm{~Hz}$ and $\sim 800 \mathrm{~Hz}$ which are common to all three sensors and are assumed to be the vibration modes of the mount. The first frequency for all but sensor $1(55 \mathrm{~Hz})$ is below the lower frequency limit of the reference accelerometer. The second natural frequencies, between 500 and $1000 \mathrm{~Hz}$ on the figure, show that as the mass on the end of the fibre cantilever is increased, this resonant frequency decreases. This is also true of the third natural frequencies. This agrees with equation (2). 


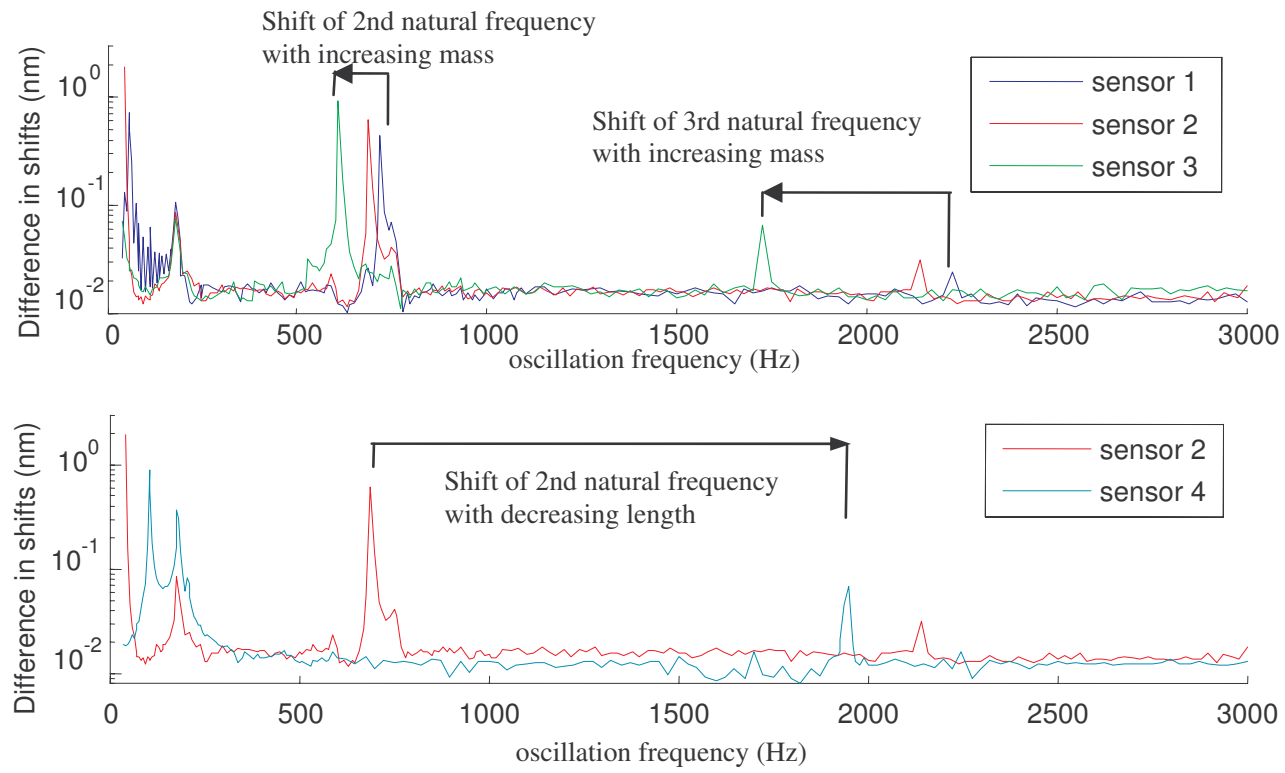

Fig 8: Comparison of frequency responses for MCF accelerometer with different masses (top) and different lengths (bottom)

Figure 8 (bottom) shows the effect of shortening the cantilever from $24.95 \mathrm{~mm}$ to $15.63 \mathrm{~mm}$. The frequency response for sensor 2 is also shown in this plot since it has the most comparable end mass to sensor 4 . The frequency shifts shown in this figure also agree with equation (2).

The MCF cantilever may be most easily used as a measurement tool in the limited frequency bands between the resonances. It is shown in figure 8 that these resonances may be tuned to give a flat response over a desired frequency range by using a suitable combination of cantilever length and end mass. The usable frequency range may be extended by using critical damping or geometries other than a simple cantilever.

\subsection{Acceleration measurement}

For several frequencies, the response of the MCF accelerometer (sensor 4) was recorded at various amplitudes of vibration. As before this was compared with the response of the reference piezoelectric accelerometer. These results are summarised is figure 9 for $30 \mathrm{~Hz}$ to $200 \mathrm{~Hz}$ and figure 10 for $300 \mathrm{~Hz}$ to $3000 \mathrm{~Hz}$. Figure 9 shows that at low frequencies, the MCF accelerometer has a linear response close to the expected value. At frequencies of $300 \mathrm{~Hz}$ and higher, the response of the MCF accelerometer, as shown in figure 10, is seen to have a varying gradient which is better represented by a quadratic rather than a linear fit. A possible explanation for this result is that at higher frequencies the fibre is vibrating in a mixture of modes which depend on amplitude.

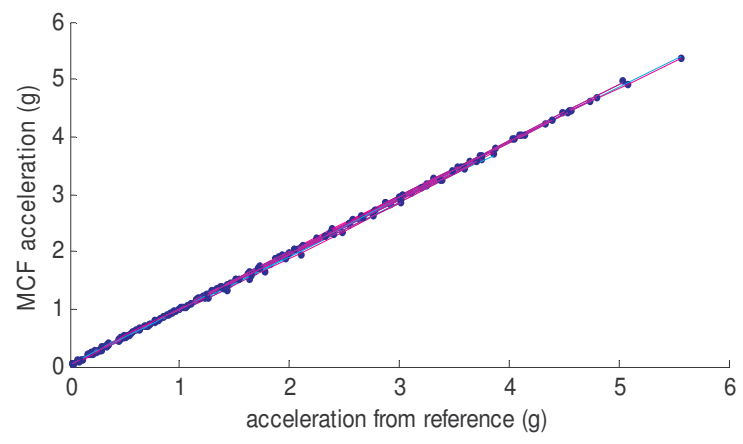

Fig 9: $\mathrm{MCF}$ acceleration vs. reference acceleration for 30 $\mathrm{Hz}, 40 \mathrm{~Hz}$ and $110 \mathrm{~Hz}-200 \mathrm{~Hz}(10 \mathrm{~Hz}$ steps $)$

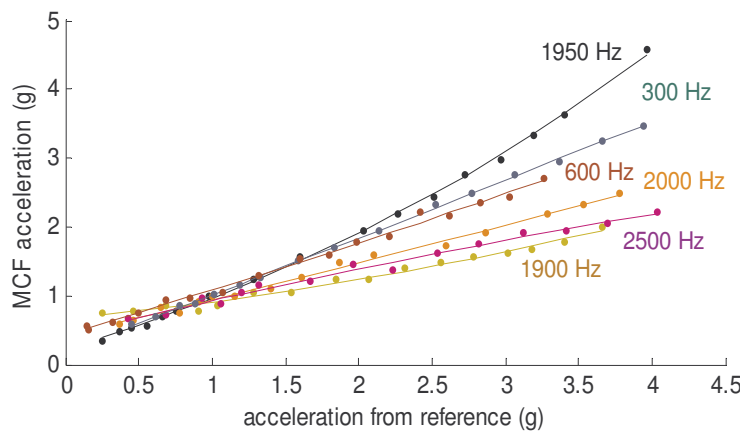

Fig 10: MCF acceleration vs. reference acceleration for selected frequencies $>200 \mathrm{~Hz}$ 
Assuming that the reference accelerometer is accurate, the gradient of each line measures the accuracy of the sensor at that frequency. Examining the gradients of these plots shows that for most frequencies below $300 \mathrm{~Hz}$, the MCF accelerometer has an accuracy of $5 \%$ or better in the acceleration range measured. Above $300 \mathrm{~Hz}$, the accuracy decreases to, in most cases, $50-60 \%$, except at $1950 \mathrm{~Hz}$, which is a resonant frequency.

\subsection{Two-axis acceleration measurement}

To demonstrate two-axis acceleration measurement, the frequency response of the sensor aligned with the vibration axis was measured. The response was then measured with the sensor rotated 90 degrees. Figure 11 shows that the responses at these two orientations match closely. Differences may arise from any asymmetry in the end mass or the housing.

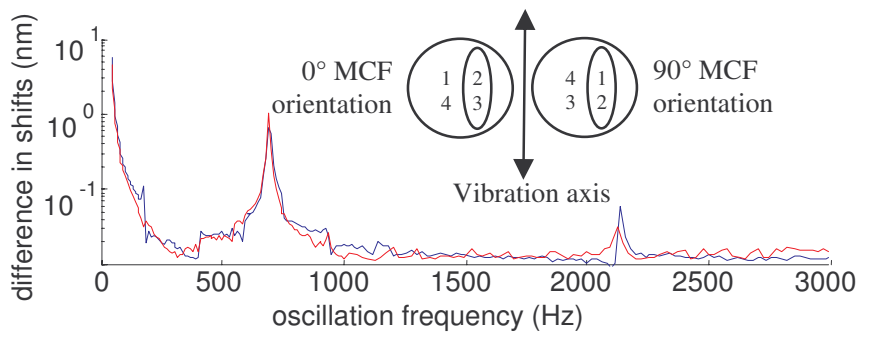

Fig 11: Vibration axis frequency response of $\mathrm{MCF}$ accelerometer at two orthogonal orientations

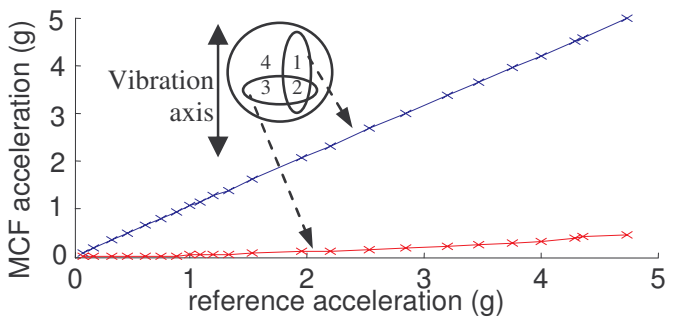

Fig 12: Vibration and cross axis response of MCF accelerometer at $40 \mathrm{~Hz}$ where shaker has low cross axis response

To show that the sensor could simultaneously measure acceleration in two axes, the vibration and cross axis responses of the MCF were recorded between 0 and $6 \mathrm{~g}$ at a vibration frequency of $40 \mathrm{~Hz}$. The results are plotted in figure 12. At this frequency, an almost zero cross axis component was measured on the shaker. The figure shows that the sensor measured a significantly higher response in the vibration axis than in the cross axis. Any rotational misalignment of the MCF would increase the measured cross axis response.

\section{CONCLUSIONS}

An all-optical accelerometer based on MCF curvature measurement with dynamic interrogation from AWGs is presented. The sensor was found to be capable of measuring acceleration in two orthogonal axes with an accuracy of at least $5 \%$ for frequencies below $300 \mathrm{~Hz}$. It was shown that the frequency response of the sensor could be adjusted by altering the length and end mass fixed to the end of the fibre cantilever which formed the sensing element.

Acknowledgements A. Fender wishes to acknowledge EPSRC and AWE plc for studentship funding. W. MacPherson wishes to acknowledge EPSRC for funding under the Advanced Research Fellowship scheme.

\section{REFERENCES}

1. A. Fender, E. J. Rigg, R. R. J. Maier, W. N. MacPherson, J. S. Barton, A. J. Moore, J. D. C. Jones, D. Zhao, L. Zhang, I. Bennion, S. McCulloch, and B. J. S. Jones, " Dynamic two-axis curvature measurement using multicore fiber Bragg gratings interrogated by arrayed waveguide gratings," Appl. Opt. 45, 9041-9048 (2006)

2. P.D. Dinev, "Two-dimensional fiber-optical accelerometer", Rev. Sci. Instrum. 67, 288-290 (1996)

3. J. M. Gere and S.P. Timoshenko, Mechanics of Materials, PWS, 1997

4. J. Kalenik and R. Pajak, "A cantilever optical-fiber accelerometer",

5. R.A.Soref and D.H.McMahon, "Tilting-mirror fiber-optic accelerometer", App. Opt. 23, 486-491 (1984)

6. J.M.Lopez-Higuera, M.A.Morante, A.Cobo, "Simple low-frequency optical fiber accelerometer", J. Lightwave Technol., 15, 1120-1130 (1997)

7. C. Z.Shi, N.Zeng, H.L.Lo, C.C.Chan, M.Zhang, W.Jin, Y.B.Liao, "Cantilever optical vibrometer using fiber Bragg grating" Opt. Eng. 42, 3179-3181 (2003)

8. T.A. Berkoff and A.D. Kersey, "Experimental demonstration of a fiber Bragg grating accelerometer", Photonics Technol. Lett. 8, 1677-1679 (1996)

9. S.J. Spammer and P.L. Fuhr, "Temperature insensitive fiber optic accelerometer using a chirped Bragg grating", Opt. Eng. 39, 2177-2181 (2000) 